\section{Morphological alterations of the tendon and pulley on ultrasound after intrasynovial injection of betamethasone for trigger digit}

\author{
Mitsuhiko Takahashi ${ }^{1,2}$, Ryosuke Sato ${ }^{2}$, Kenji Kondo ${ }^{2}$, Koichi Sairyo $^{2}$ \\ 'Department of Orthopaedics, Tokushima Prefectural Central Hospital, Tokushima; \\ ${ }^{2}$ Department of Orthopaedics, Tokushima University Graduate School of Biomedical Science, \\ Tokushima, Japan
}

Purpose: The aim of this study was to elucidate whether intrasynovial corticosteroid injections for trigger digit reduced the volume of the tendon and pulley on high-resolution ultrasonography. Methods: Twenty-three digits of 20 patients with trigger digit were included. Each affected finger was graded clinically according to the following classification: grade I for pre-triggering, grade II for active triggering, grade III for passive triggering, and grade IV for presence of contracture. Axial ultrasound examinations were performed before an intrasynovial corticosteroid injection and at an average of 31 days after the injection. The transverse diameter, thickness, and crosssectional area of the tendon and the thickness of the pulley were measured by two independent, blinded researchers.

Results: At least 1 grade of improvement was achieved in this study group by the time of the second examination. The transverse diameter and cross-sectional area of the tendon and the thickness of the pulley significantly decreased $(P<0.05)$.

Conclusion: The injection of a single dose of betamethasone improved clinical symptoms by reducing the volume of both the tendon and pulley, which may be related to the fact that tendon and pulley ruptures are delayed by corticosteroid injections.

Keywords: Trigger finger disorder; Ultrasound; Tendons; Adrenal cortex hormones

\section{Introduction}

Although trigger digit is a relatively common complaint in orthopaedic clinics, its cause is not fully understood. The main symptoms are snapping of the digit and tenderness over the first annular (A1) pulley. It is thought to be caused by stenosing tenosynovitis or tendon entrapment at the A1 pulley induced by a mismatch between the size of the pulley and the volume of the flexor tendons [1].

Many cases of symptomatic trigger digit can now be managed non-surgically with corticosteroid injections, which have been found to be effective in $64 \%-97 \%$ of cases [2-7]. However, postinjection changes in the pulley and tendon have not been fully investigated. Moreover, concerns
ULTRA

SONO

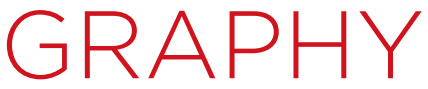

ORIGINAL ARTICLE

https://doi.org/10.14366/usg. 17038 pISSN: 2288-5919 - elSSN: 2288-5943 Ultrasonography 2018;37:134-139

Received: May 11, 2017

Revised: July 21, 2017

Accepted: July 25, 2017

Correspondence to: Mitsuhiko Takahashi, MD, PhD, Department of Orthopaedics, Tokushima Prefectural Central Hospital, 1-10-3, Kuramoto, Tokushima 770-8539, Japan

Tel. +81-88-631-7151

Fax. +81-88-631-8354

E-mail:w-tk@umin.ac.jp

This is an Open Access article distributed under the terms of the Creative Commons Attribution NonCommercial License (http://creativecommons.org/ licenses/by-nc/3.0/) which permits unrestricted noncommercial use, distribution, and reproduction in any medium, provided the original work is properly cited.

Copyright @ 2018 Korean Society of Ultrasound in Medicine (KSUM)

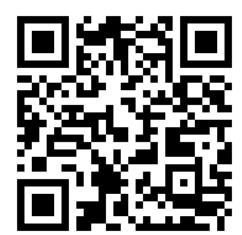

How to cite this article:

Takahashi M, Sato R, Kondo K, Sairyo K. Morphological alterations of the tendon and pulley on ultrasound after intrasynovial injection of betamethasone for trigger digit Ultrasonography. 2018 Apr;37(2):134-139. 
have been raised about the deteriorative effect of corticosteroids on connective tissue.

Recently, high-resolution ultrasonography with a high-frequency transducer has enabled morphological changes in small structures in the finger to be observed [8]. Serafini et al. [9] reported that flexor tendon thickening was the main cause of trigger digit and suggested that a reasonable cut-off for pathological findings was a $20 \%$ increase in tendon thickness compared with the contralateral tendon. Guerini et al. [10] reported thickening and hypoechogenicity of the A1 pulley in all patients with trigger digit, and hypervascularity of the A1 pulley in $91 \%$ of patients. In addition, Sato et al. [11] reported that the tendon tended to be thicker under the A1 pulley in accordance with the clinical grade of trigger digit, and that thickening of the volar plate affected continuous triggering. Although several pathophysiological mechanisms have been suggested, trigger digit can be reliably assessed by ultrasonographic measurements of the pulley and tendon.

Hence, this study employed high-resolution ultrasonography to investigate morphological changes in the tendon and the pulley after a single corticosteroid (betamethasone suspension) injection in patients with trigger digit.

\section{Materials and Methods}

All study procedures were conducted in accordance with the 1975 Declaration of Helsinki, as revised in 2008 [12], and this study received approval from the Institutional Review Board. Informed consent to be included in this study was obtained from all patients. Consecutive patients clinically diagnosed with trigger digit at our institution between April 2011 and March 2015 were recruited, while patients with rheumatoid arthritis, a prior local corticosteroid injection, severe diabetes mellitus meaning that a local corticosteroid injection might be harmful, a history of infectious disease or major trauma in the same digit, or who were unwilling to participate in this study were excluded from this study. We used radiographs to confirm that none of the patients had a bony deformity, calcium deposition, or osteoarthritis in the digit. Twenty-three digits (five thumbs, one index, nine middle, and eight ring fingers) of 20 patients (seven males and 13 females; mean age, $64.0 \pm 9.7$ years; range, 44 to 86 years) with trigger digit were ultimately included in this study after the exclusion of a patient who did not show clinical improvement after the treatment. Each affected finger was graded clinically according to signs of triggering: grade I (pretriggering) was defined by pain and a history of catching, but without demonstrable catching on the physical examination; grade II (active triggering) involved demonstrable catching, but the ability to actively extend the digit; grade III (passive triggering) was assigned to patients requiring passive extension or presenting an inability to engage in active flexion; and grade IV (contracture) was assigned to patients who showed fixed flexion contracture of the proximal interphalangeal joint [13].

All ultrasound examinations and patient management were performed by one author (M.T., 11 years of experience of hand surgery and $>20$ years of orthopaedic surgery). An axial view using high-resolution ultrasonography (EUB-7500 with a linear-array high-frequency transducer, Hitachi Medical Corp., Tokyo, Japan) was obtained from the volar aspect at the metacarpophalangeal (MP) joint with minimum pressure. The patient was relaxed in the supine position with the affected forearm positioned in supination, the wrist in neutral, and the MP joint in extension. The transducer was placed perpendicular to the tendon, such that the tendon was characterised by its hyperechoic fibrillar pattern and the surrounding fluid distension was maximal. After confirming thickening of the A1 pulley in comparison with that of the adjacent non-affected digit or that of the contralateral digit, $2.5 \mathrm{mg}(0.5 \mathrm{~mL})$ of an injectable suspension of betamethasone (Rinderon, Shionogi Co. Ltd., Osaka, Japan) with $10 \mathrm{mg}(0.5 \mathrm{~mL})$ of procaine was administered into the intrasynovial space beneath the affected pulley. The injection was made with a 27-gauge needle in a relief-of-resistance manner and with palpation of the distal tendon sheath to monitor ballooning during the injection. Patients were advised to exercise the affected digit after the injection. An ultrasound examination was repeated in the same manner as the initial examination at an average of 31 days (range, 26 to 39 days) after the injection. The axial images presenting maximal surrounding fluid distension both before and after the injection were chosen by the examiner (M.T.) for later measurements (Fig. 1). Two independent raters, both with $>8$ years of experience of orthopaedic surgery, who were blinded to all medical information about the patients, measured the selected parameters on the chosen image. The transverse diameter, thickness, and cross-sectional area of the tendon and the thickness of the pulley were measured (Fig. 2). The two raters measured these parameters on an independent computer with ImageJ software (ver. 1.46r, NIH, Bethesda, MD, USA) at almost the same time. Statistical analyses were performed using SPSS Statistics for Windows, ver. 22.0 (IBM Corp., Armonk, NY, USA). Linear regression was performed and intraclass correlation coefficients (ICCS) were calculated to quantify the level of agreement between the two raters. All data are averages from the two raters. Data are expressed as mean and standard error of the mean, unless otherwise mentioned. The paired $t$ test was used to compare findings from the two ultrasound examinations. P-values $<0.05$ were considered to indicate statistical significance. 


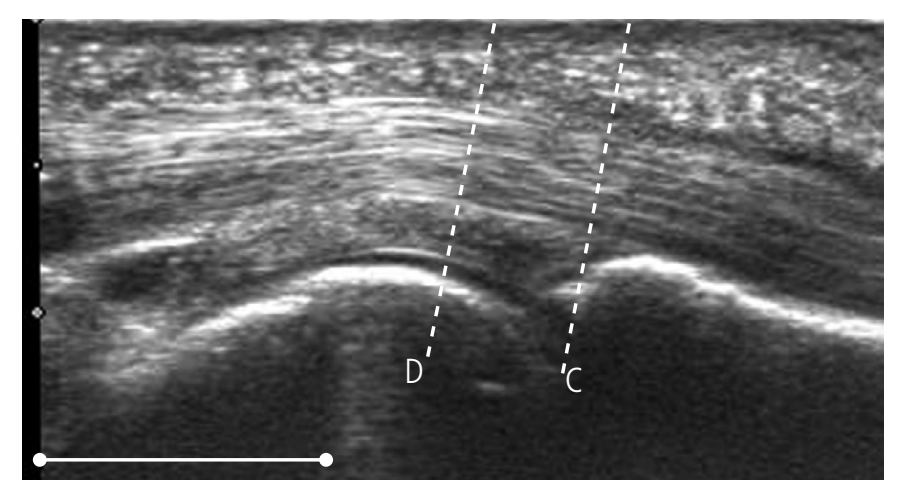

A

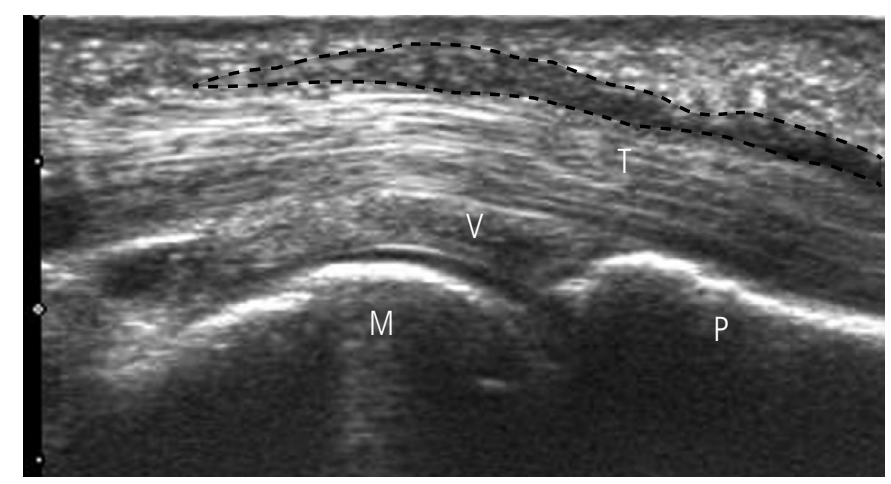

B

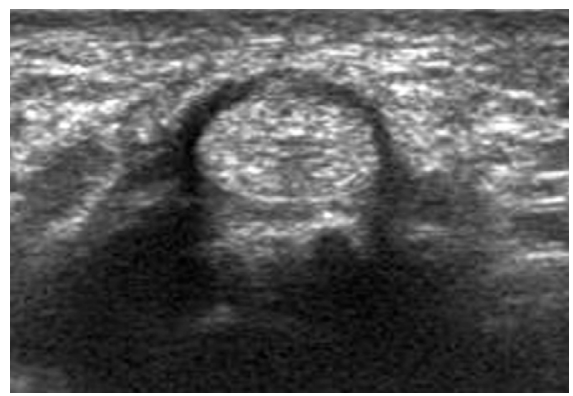

C

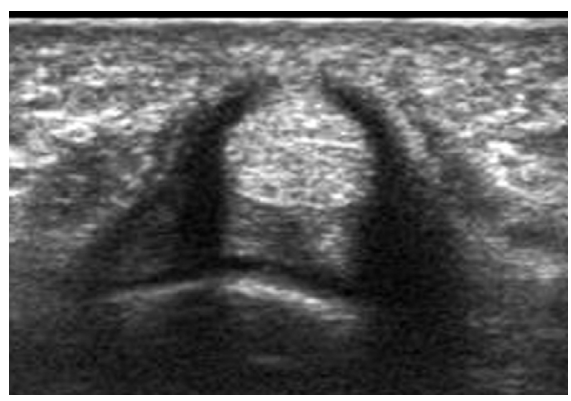

D

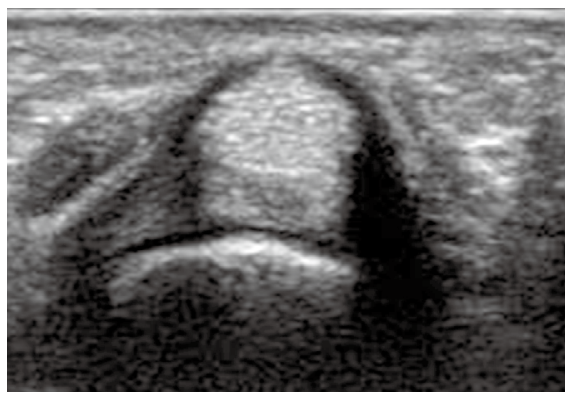

$\mathrm{E}$

Fig. 1. A 70-year-old man with grade III trigger finger at the left 4th finger.

A. Longitudinal view on ultrasonography over the palmar aspect of the affected metacarpophalangeal joint refers the positional relation of the axial images. Dotted lines indicate the corresponding axial planes shown in $C$ and $D$. Scale bar $=10 \mathrm{~mm}$. B. The same view with A shows the $A 1$ and $A 2$ pulleys (shaded area) over the tendon $(T)$, volar plate $(V)$, metacarpal head $(M)$ and proximal phalanx $(P)$. C, D. Consecutive axial images show the distal, less thick pulley (C) and the thickest pulley (D). Hypoechoic fluid distension was maximal at the level of D, E. Ultrasonography at the same level as D conducted 26 days after the injection shows decreased thickness of the tendon (from 3.78 to 3.41 $\mathrm{mm}$ ), cross-sectional area of the tendon (from 18.52 to $16.15 \mathrm{~mm}^{2}$ ), and thickness of the pulley (from 0.922 to $0.533 \mathrm{~mm}$ ), but no decrease in the transverse diameter of the tendon (from 5.59 to $5.81 \mathrm{~mm}$ ) in this case.

\section{Results}

In this study population, two digits were grade II, 19 were grade III, and two were grade IV prior to the injection. At the second ultrasound examination, at least one grade of improvement was achieved in this study group: 14 digits were grade I and nine were grade II. Thus, overall improvement to the point that triggering was absent occurred in $61 \%$ of the population (14 of 23 digits). No patients complained of any side effects after the injection. All measurement values obtained from the two raters agreed well. This was reflected in the results of regression analyses showing good inter-rater agreement $\left(n=23 ; P<0.001\right.$ for all parameters; $r^{2}=0.853-$ 0.965). Absolute numeric agreement was excellent (ICC, 2, 1; range, 0.899 to 0.980 ), confirming the validity of the measurements made using ImageJ.

After the injection, the transverse diameter and cross-sectional area of the tendon and the thickness of the pulley significantly decreased, as assessed by both raters $(P<0.05$ for these three 


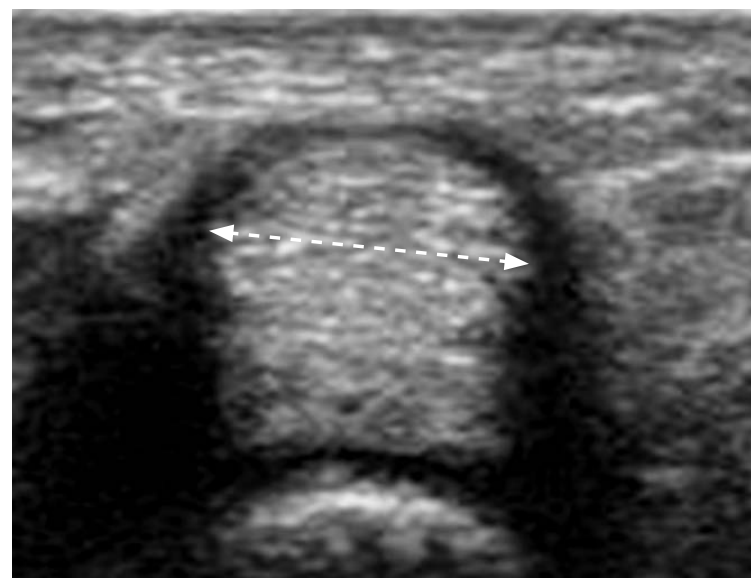

A

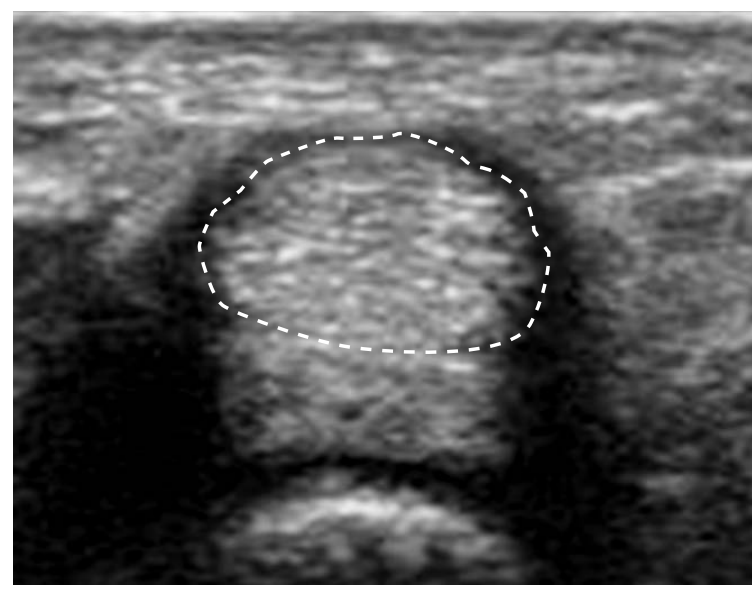

C

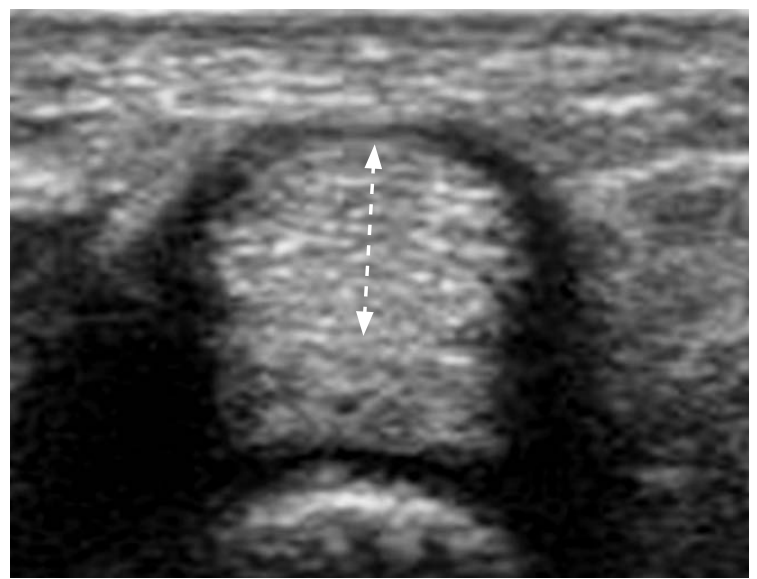

B

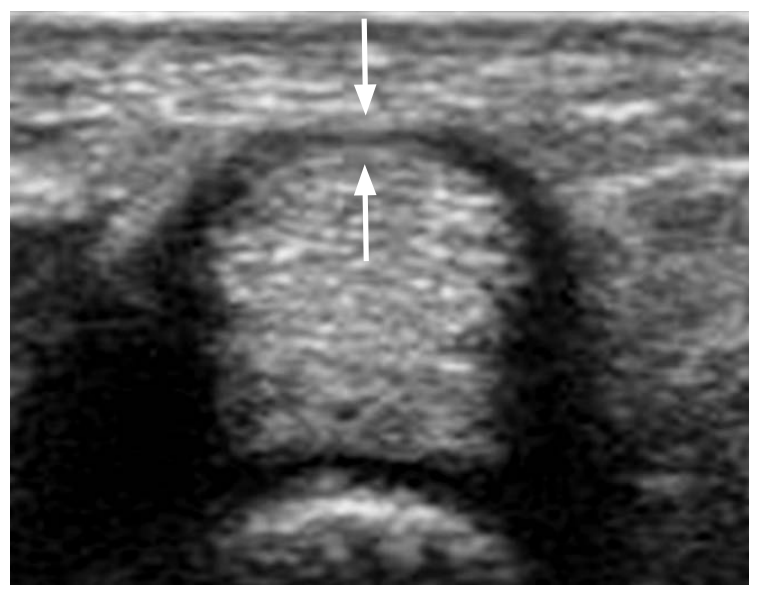

D

Fig. 2. Axial view for the measurements.

A-D. Axial view on ultrasonography is used in this study to measure four parameters: the transverse diameter of the tendon ( $A$, dotted line), the thickness of the tendon ( $B$, dotted line), the cross-sectional area of the tendon $\left(C\right.$, dotted line), and the thickness of the pulley $\left(D_{\text {, }}\right.$ arrows). The tendon shows a hyperechoic fibrillar pattern, while hypoechoic fluid distension is maximal.

Table 1. Ultrasound parameters measured by the two independent raters before and after the injection

\begin{tabular}{|c|c|c|c|}
\hline Parameter & Before injection & After injection & P-value \\
\hline \multicolumn{4}{|c|}{ Transverse diameter of the tendon (mm) } \\
\hline Rater 1 & $6.29 \pm 0.20$ & $5.93 \pm 0.20$ & 0.011 \\
\hline Rater 2 & $6.27 \pm 0.20$ & $5.74 \pm 0.19$ & 0.009 \\
\hline \multicolumn{4}{|l|}{ Thickness of the tendon (mm) } \\
\hline Rater 1 & $4.52 \pm 0.16$ & $4.38 \pm 0.12$ & 0.119 \\
\hline Rater 2 & $4.72 \pm 0.17$ & $4.53 \pm 0.15$ & 0.072 \\
\hline \multicolumn{4}{|c|}{ Cross-sectional area of the tendon $\left(\mathrm{mm}^{2}\right)$} \\
\hline Rater 1 & $21.67 \pm 1.34$ & $19.01 \pm 1.15$ & 0.020 \\
\hline Rater 2 & $22.34 \pm 1.44$ & $19.70 \pm 1.28$ & 0.016 \\
\hline \multicolumn{4}{|l|}{ Thickness of the pulley (mm) } \\
\hline Rater 1 & $1.12 \pm 0.10$ & $0.70 \pm 0.05$ & 0.002 \\
\hline Rater 2 & $1.06 \pm 0.09$ & $0.67 \pm 0.04$ & $<0.001$ \\
\hline
\end{tabular}


parameters) (Table 1). The thickness of the tendon also decreased after the injection, although it was not significant, according to both raters $(P>0.05)$.

\section{Discussion}

This study demonstrated that measured values of tendon volume and pulley thickness decreased approximately 1 month after a single betamethasone injection into the intrasynovial space of symptomatic tendons and pulleys. The thickness of the pulley is usually quite small ( $<1 \mathrm{~mm}$ for a normal pulley). The recent development of highresolution ultrasonography with a high-frequency transducer has enabled the detection of subtle changes in the thickness of the pulley.

Our results regarding changes in thickness of the pulley after betamethasone injection largely concur with those reported by Miyamoto et al. [14], who reported that increased thickness and stiffness of the A1 pulley were causes of snapping in the trigger digit, and that the thickness and stiffness of the A1 pulley decreased 3 weeks after triamcinolone acetonide injection. Their report, however, reported no reduction of the cross-sectional area of the tendon after the injection. This divergence in the results regarding the cross-sectional area of the tendon may be due to differences between the studies, including the corticosteroid that was applied and the timing of the ultrasound examination. In the previous study, the researchers applied $10 \mathrm{mg}$ of triamcinolone acetonide to the tendon sheath, whereas we used $2.5 \mathrm{mg}$ of betamethasone, which is 5- to 6-fold more potent per milligram in terms of its antiinflammatory effects than triamcinolone. The second ultrasound examination was performed 3 weeks after the injection in the previous study, whereas we performed the second examination at an average of 1 month after the injection.

The therapeutic effects of a corticosteroid injection into the trigger digit may vary. Reduction of the volume might be achieved through a decrease in tissue oedema, which is a pharmacological effect of corticosteroids $[15,16]$. Several experimental studies have shown that corticosteroid-induced tendinopathies occurred through reduced synthesis of collagen type I and proteoglycans, as well as decreased tenocyte proliferation, differentiation, viability, and metabolism [17-22]. It has also been shown that under specific conditions, corticosteroids increase the synthesis of matrix metalloproteinase (MMP)-1 and MMP-13, both of which can cleave collagen type I $[23,24]$. Considering these findings, it appears that corticosteroids may negatively affect the tendon and the pulley, although the corticosteroid dosage and the timing of the examination should be considered.

Despite the controversy over the effects of corticosteroids on the tendon, it is certain that reduction of the pulley thickness is a major mechanism through which corticosteroid injections affect trigger digit. In contrast to the tendon, which consists of a dense collagen matrix, the pulley has been shown to have a loose connective layer in addition to a dense normal connective layer [25]. In addition, an innermost layer composed of irregular connective tissue has been reported in pathological conditions such as trigger digit. These less dense elements are thought to be more susceptible to corticosteroids than dense tissues, such as tendons. We speculate that the effects of corticosteroids on the tendons emerge later than the effects on the pulleys due to the difference in the density of the connective tissue.

Delayed ruptures of the flexor tendon or pulley after a corticosteroid injection have been reported in the literature [26-29]. In these reports, multiple corticosteroid injections were administered, and the rupture occurred many months after the first injection. Even though a causative link between corticosteroid injection and tendon or pulley rupture cannot be made, all authors have recommended that repetitive intratendinous or intrasynovial injections should be avoided due to the known attritional effects of corticosteroids on collagen. These reports indicate that the deteriorative effects of corticosteroid on the tendon last for a long period, and that careful observation is required for many months after corticosteroid injection.

This study has several limitations. First, the sample size was relatively small, although we addressed a commonly occurring pathological condition. However, the design of the study, in which two independent, blinded raters measured the ultrasound parameters, may compensate for the small sample size. Second, this study did not have a control group. Since corticosteroid injection for trigger digit is a widely accepted treatment and the patients came to our institution seeking treatment with a definitive effect, we did not design a placebo control group. Finally, we did not examine the parameters on ultrasound at a later time point, which would be another issue of interest. A goal of our future research is to address the long-term effects of betamethasone injections.

In summary, we have shown, using axial high-resolution ultrasonography, that a single dose of intrasynovial betamethasone injection for trigger digit improved clinical symptoms by reducing not only the thickness of the pulley, but also the volume of the tendon. Further studies are needed to understand the deteriorative effects of corticosteroids on tendinous tissue and to identify safe doses for corticosteroid injections.

ORCID: Mitsuhiko Takahashi: http://orcid.org/0000-0002-8134-1098; Ryosuke Sato: http://orcid.org/0000-0003-3985-2721; Kenji Kondo: http://orcid.org/0000-00024073-1815; Koichi Sairyo: http://orcid.org/0000-0003-3794-5369 


\section{Conflict of Interest}

No potential conflict of interest relevant to this article was reported.

\section{References}

1. Ryzewicz M, Wolf JM. Trigger digits: principles, management, and complications. J Hand Surg Am 2006;31:135-146.

2. Kolind-Sorensen V. Treatment of trigger fingers. Acta Orthop Scand 1970;41:428-432.

3. Rhoades CE, Gelberman RH, Manjarris JF. Stenosing tenosynovitis of the fingers and thumb. Results of a prospective trial of steroid injection and splinting. Clin Orthop Relat Res 1984;(190):236-238.

4. Freiberg A, Mulholland RS, Levine R. Nonoperative treatment of trigger fingers and thumbs. J Hand Surg Am 1989;14:553-558.

5. Newport ML, Lane LB, Stuchin SA. Treatment of trigger finger by steroid injection. J Hand Surg Am 1990;15:748-750.

6. Murphy D, Failla JM, Koniuch MP. Steroid versus placebo injection for trigger finger. J Hand Surg Am 1995;20:628-631.

7. Salim N, Abdullah S, Sapuan J, Haflah NH. Outcome of corticosteroid injection versus physiotherapy in the treatment of mild trigger fingers. J Hand Surg Eur Vol 2012;37:27-34.

8. Lee SA, Kim BH, Kim SJ, Kim JN, Park SY, Choi K. Current status of ultrasonography of the finger. Ultrasonography 2016;35:110-123.

9. Serafini G, Derchi LE, Quadri P, Martinoli C, Orio O, Cavallo A, et al. High resolution sonography of the flexor tendons in trigger fingers. J Ultrasound Med 1996;15:213-219.

10. Guerini H, Pessis E, Theumann N, Le Quintrec JS, Campagna R, Chevrot $A$, et al. Sonographic appearance of trigger fingers. J Ultrasound Med 2008;27:1407-1413.

11. Sato J, Ishii Y, Noguchi H, Takeda M. Sonographic appearance of the flexor tendon, volar plate, and $\mathrm{A} 1$ pulley with respect to the severity of trigger finger. J Hand Surg Am 2012;37:2012-2020.

12. Williams JR. The Declaration of Helsinki and public health. Bull World Health Organ 2008;86:650-652.

13. Wolfe SW. Tendinopathy. In: Wolfe SW, Hotchkiss RN, Pederson WC, Kozin SH, eds. Green's operative hand surgery. 6th ed. Philadelphia, PA: Elsevier/Churchill Livingston, 2011;2068-2088.

14. Miyamoto H, Miura T, Isayama $H$, Masuzaki R, Koike K, Ohe T. Stiffness of the first annular pulley in normal and trigger fingers. J Hand Surg Am 2011;36:1486-1491.

15. Forster C, Silwedel C, Golenhofen N, Burek M, Kietz S, Mankertz $J$, et al. Occludin as direct target for glucocorticoid-induced improvement of blood-brain barrier properties in a murine in vitro system. J Physiol 2005;565(Pt 2):475-486.

16. Heiss JD, Papavassiliou E, Merrill MJ, Nieman L, Knightly JJ, Walbridge $S$, et al. Mechanism of dexamethasone suppression of brain tumor-associated vascular permeability in rats: involvement of the glucocorticoid receptor and vascular permeability factor. I Clin Invest 1996;98:1400-1408.

17. Torricelli P, Fini M, Giavaresi G, Carpi A, Nicolini A, Giardino R. Effects of systemic glucocorticoid administration on tenocytes. Biomed Pharmacother 2006;60:380-385.

18. Wong MW, Tang YN, Fu SC, Lee KM, Chan KM. Triamcinolone suppresses human tenocyte cellular activity and collagen synthesis. Clin Orthop Relat Res 2004;(421):277-281.

19. Wong MW, Tang YY, Lee SK, Fu BS, Chan BP, Chan CK. Effect of dexamethasone on cultured human tenocytes and its reversibility by platelet-derived growth factor. J Bone Joint Surg Am 2003:85:1914-1920.

20. Wong MW, Tang YY, Lee SK, Fu BS. Glucocorticoids suppress proteoglycan production by human tenocytes. Acta Orthop 2005;76:927-931.

21. Niessen FB, Spauwen PH, Schalkwijk J, Kon M. On the nature of hypertrophic scars and keloids: a review. Plast Reconstr Surg 1999; 104:1435-1458.

22. Chen W, Tang H, Zhou M, Hu C, Zhang J, Tang K. Dexamethasone inhibits the differentiation of rat tendon stem cells into tenocytes by targeting the scleraxis gene. J Steroid Biochem Mol Biol 2015;152:16-24.

23. Sadowski T, Steinmeyer J. Effects of non-steroidal antiinflammatory drugs and dexamethasone on the activity and expression of matrix metalloproteinase-1, matrix metalloproteinase- 3 and tissue inhibitor of metalloproteinases- 1 by bovine articular chondrocytes. Osteoarthritis Cartilage 2001;9:407-415.

24. Sendzik J, Shakibaei M, Schafer-Korting M, Lode H, Stahlmann R. Synergistic effects of dexamethasone and quinolones on humanderived tendon cells. Int J Antimicrob Agents 2010;35:366-374.

25. Sbernardori MC, Bandiera P. Histopathology of the A1 pulley in adult trigger fingers. J Hand Surg Eur Vol 2007;32:556-559.

26. Taras JS, liams GJ, Gibbons M, Culp RW. Flexor pollicis longus rupture in a trigger thumb: a case report. J Hand Surg Am 1995:20:276-277.

27. Fitzgerald BT, Hofmeister EP, Fan RA, Thompson MA. Delayed flexor digitorum superficialis and profundus ruptures in a trigger finger after a steroid injection: a case report. J Hand Surg Am 2005;30:479-482.

28. Gyuricza C, Umoh E, Wolfe SW. Multiple pulley rupture following corticosteroid injection for trigger digit: case report. J Hand Surg Am 2009;34:1444-1448.

29. Nguyen ML, Jones NF. Rupture of both the abductor pollicis longus and extensor pollicis brevis tendons after steroid injection for de quervain tenosynovitis. Plast Reconstr Surg 2012;129:883e-886e. 